\title{
Coalition Formation Under Dominance Invariance
}

\author{
Mert Kimya ${ }^{1}$
}

Accepted: 4 February 2022

(c) The Author(s) 2022

\begin{abstract}
An abstract game satisfies Dominance Invariance if the indirect and the direct dominance relations, or myopic and farsighted dominance, are equivalent. Mauleon et al. (Int J Game Theory 43(4):925-943, 2014) study Dominance Invariance as an attractive condition that eliminates the differences between a farsighted solution concept and its myopic counterpart. We show that Dominance Invariance can also be used to eliminate the differences between various farsighted solution concepts in any abstract game. Together with an additional condition called No Infinite Chains, Dominance Invariance implies the existence and uniqueness of the farsighted stable set, its equivalence to the largest consistent set and its equivalence to the (strong) rational expectations farsighted stable set when the latter exists. This also implies that both the farsighted stable set and the largest consistent set do not suffer from the problem of maximality under these conditions.
\end{abstract}

Keywords Farsighted stability $\cdot$ Coalitional games $\cdot$ Farsighted stable set $\cdot$ Largest consistent set

JEL Classification C70 $\cdot$ C71 $\cdot$ D70 $\cdot$ D71

\section{Introduction}

Dominance Invariance refers to the equivalence of the direct and the indirect dominance relations. It was analyzed in matching problems by Mauleon et al. [25] as an attractive condition that eliminates the differences between a farsighted solution concept and its myopic counterpart.

Can we take this a step further and use Dominance Invariance to also eliminate the differences between different farsighted solution concepts? This paper shows that if we combine

This article is part of the topical collection "Group Formation and Farsightedness" edited by Francis Bloch, Ana Mauleon and Vincent Vannetelbosch.

I thank an anonymous referee for helpful comments and suggestions. I gratefully acknowledge financial support from Australian Research Council DE210101085 grant.

$凶$ Mert Kimya

mert.kimya@sydney.edu.au

1 School of Economics, University of Sydney, Camperdown, Sydney, NSW 2006, Australia 
Dominance Invariance with a condition called No Infinite Chains, then we can achieve this to a great extent. Specifically, we show that in any abstract game that satisfies these two conditions: (i) there exists a unique farsighted stable set, (ii) this unique farsighted stable set is equal to the largest consistent set, and (iii) these solution concepts do not suffer from the problem of maximality as they are also equivalent to the (strong) rational expectations farsighted stable set $[(\mathrm{S}) \mathrm{REFS}]$, when the latter exists. We further provide an algorithm to find the unique farsighted stable set when the game is finite and show examples of classes of games that satisfy Dominance Invariance and No Infinite Chains.

Harsanyi [13] criticized the stable set of von Neumann and Morgenstern [32] for being myopic. To overcome this criticism, Chwe [8] formalized the notion of an indirect dominance relation, which is the farsighted counterpart of the direct dominance relation myopic solution concepts are defined with. ${ }^{1}$ Using this notion of indirect dominance, Chwe [8] defined the solution concept of the largest consistent set and also analyzed the notion of the stable set by replacing the direct dominance with the indirect dominance relation; the latter is known as the farsighted stable set.

Both the farsighted stable set and the largest consistent set have become highly influential solution concepts, ${ }^{2}$ but they have also been criticized for not embodying the idea of maximality (see Ray and Vohra [28]). This refers to the observation that instead of considering the optimal course of action, in these concepts players form extreme expectations based on optimism or pessimism leading to unreasonable predictions in some situations (see Xue [35] and Ray and Vohra [28]). There is a growing literature that tries to incorporate this notion of maximality into the farsighted solution concepts. For examples, see Dutta and Vohra [11], Dutta and Vartiainen [10], Herings et al. [14], Karos and Robles [19], Kimya [20], and Konishi and Ray [23].

In this paper, we take a complementary approach and find conditions under which the 'optimistic' solution concept of the farsighted stable set coincides with the 'pessimistic' solution concept of the largest consistent set and consequently they also do not suffer from the problem of maximality.

One of these conditions and our starting point is Dominance Invariance analyzed in Mauleon et al. [25] in matching problems. They make the observation that under Dominance Invariance the level of foresight the individuals possess does not matter as the farsighted solution concepts coincide with their myopic counterparts. This observation is valuable given the evidence for differing levels of foresight individuals possess (see Kirchsteiger et al. [22]).

Dominance Invariance itself is not sufficient to achieve equivalence, one can still find games that satisfy Dominance Invariance in which the farsighted stable set and the largest consistent set make different predictions. One can even find games that satisfy Dominance Invariance, but in which no farsighted stable set exists (see Sect. 3 for an example). The additional condition we introduce is No Infinite Chains, which requires the game to possess no infinite chain of direct dominance.

Proposition 1 shows that in abstract games that satisfy Dominance Invariance and No Infinite Chains a unique farsighted stable set exists that is also equivalent to the largest consistent set. Note that in general not only the largest consistent set and the farsighted stable set differ, but also a farsighted stable set need not exist and when it does it need not be unique.

\footnotetext{
1 An indirect dominance relation was also used in Harsanyi [13], but this definition is different. Chwe's [8] definition is the one that became standard in the literature.

2 For example see Diamantoudi and Xue [9], Ray and Vohra [29, 30] for applications to characteristic function games, Herings et al. [15, 17], Cai and Kimya [7], Page et al. [26] for applications to network formation, and Bloch and van den Nouweland [6], Herings et al. [18], Kimya [21], Mauleon et al. [24] for applications to matching.
} 
Chwe [8] has already provided an algorithm that can be used to find the largest consistent set when the game is finite, we provide a significantly simpler algorithm in Proposition 2 that can be utilized to find both solution concepts under Dominance Invariance and No Infinite Chains.

In Sect. 3.3, we uncover a further advantage of games that satisfy these conditions. We show that whenever Dominance Invariance and No Infinite Chains are satisfied the farsighted stable set and the largest consistent set do not suffer from the problem of maximality. We demonstrate this by showing that under Dominance Invariance and No Infinite Chains, these solution concepts are also equivalent to the (S)REFS, when the latter exists. (S)REFS was defined in Dutta and Vohra [11] explicitly for the purpose of dealing with the problem of maximality.

Given the strong implications of these conditions, it is no surprise that these are strong conditions and sometimes they are hard to check. To demonstrate that there is a variety of situations that can be modeled with games that satisfy these conditions, we provide two simple classes of games that satisfy each condition. The first class is finite acyclic games in which No Infinite Chains condition is trivially satisfied, and one only needs to check Dominance Invariance. The second class of games is what we call beeline games. In beeline games if a coalition can initiate a path between two states, then the coalition can also directly move between these two states. In beeline games, Dominance Invariance is satisfied and one only needs to check No Infinite Chains. From the literature, we provide examples of situations that belong to each class and that satisfy both of these conditions, implying that all of our results also apply in these situations.

Although these examples point to the usefulness of these conditions, one should still keep in mind that these are very strong conditions in certain environments. To demonstrate this, we take Transferable Utility Games (TU Games) and show that only trivial TU Games satisfy Dominance Invariance. This is in stark contrast to Weber's [33] characterization of absolutely stable games, which are games in which Harsanyi's [13] indirect dominance relation, as opposed to Chwe's [8], is equivalent to direct dominance.

Finally, it should also be noted that although Dominance Invariance and No Infinite Chains lead to equivalence between prominent farsighted solution concepts, they do not completely eliminate the multiplicity of farsighted solution concepts in the literature. We demonstrate this point through DEM farsighted stable sets (see Herings et al. [15, 16]). We show that under Dominance Invariance and No Infinite Chains the unique farsighted stable set is a DEM farsighted stable set, but there might still exists other DEM farsighted stable sets.

We start by setting up the model and defining the solution concepts in the next section. Section 3 contains the main results. In Sect. 4, we analyze finite acyclic games and beeline games. Section 5 concludes with two remarks.

\section{Preliminaries}

An abstract game is defined as $\Gamma=\left\{N, Z,\left\{\succeq_{i}\right\}_{i \in N},\{\stackrel{S}{\rightarrow}\}_{S \subseteq N, S \neq \emptyset}\right\}$ (see Chwe [8], Rosenthal [31] and Xue [35]), where $N$ is the finite set of players, $Z$ is the set of states, $\left\{\succeq_{i}\right\}$ is player $i$ 's preference defined on the set of states, and $\{\stackrel{S}{\rightarrow}\}_{S \subseteq N, S \neq \emptyset}$ are effectiveness relations defined on the set of states. The effectiveness relation $\{\stackrel{S}{\rightarrow}\}$ describes what coalition $S$ can do at every state. That is, $a \stackrel{S}{\rightarrow} b$ for $a, b \in Z$ if and only if when $a$ is the status quo, coalition $S$ can change state $a$ with state $b$. 
Dominance relation is used to define the well-known solution concepts of the core and the stable set. For any coalition $S \subseteq N$, let $b \succ_{S} a$ if $b \succ_{i} a$ for all $i \in S$.

Definition 1 (Dominance) We say that $a \in Z$ is dominated by $b \in Z$, denoted $b>a$, if there exists a coalition $S \subseteq N$ such that $a \stackrel{S}{\rightarrow} b$ and $b \succ_{S} a .^{3}$

Harsanyi's [13] criticism of the myopia within the stable set led to the introduction of the indirect dominance relation (see Chwe [8], Harsanyi [13]) to incorporate farsightedness in cooperative games.

Definition 2 (Indirect Dominance) We say $a \in Z$ is indirectly dominated by $b \in Z$, denoted $b \gg a$, if there exists a sequence of states $a_{0}, a_{1}, \ldots, a_{m}$, where $a_{0}=a$ and $a_{m}=b$, and a sequence of coalitions $S_{0}, S_{1}, \ldots, S_{m-1}$ such that $a_{i} \stackrel{S_{i}}{\rightarrow} a_{i+1}$ and $b \succ_{S_{i}} a_{i}$ for $i=0,1, \ldots, m-1$.

If $b$ indirectly dominates $a$, then there exists a path from $a$ to $b$ such that every coalition effective on this path prefers state $b$, which is the final state on the path, to the state they replace. There is a number of solution concepts with various advantages and disadvantages that is defined using this indirect dominance relation (see Ray and Vohra [28] for an overview). In this paper, we will focus on the farsighted stable set and the largest consistent set (Chwe [8]). We define each of these solution concepts in turn.

Definition 3 [Largest Consistent Set (Chwe [8])] A set $V \subseteq Z$ is consistent if $a \in V$ if and only if for all $d \in Z, S \subseteq N$ such that $a \stackrel{S}{\rightarrow} d$, there exists $e \in V$, where $d=e$ or $e \gg d$, such that $e \nsucc_{S} a$. The largest consistent set is a consistent set that contains all other consistent sets. 4

Roughly, a set of states $V$ is a consistent set if a state is in $V$ if and only if any deviation from this state can be followed by an indirect dominance path back to a state in $V$ that makes at least one of the deviators weakly worse off. The largest consistent set is the unique consistent set that contains all other consistent sets. Largest consistent set is a weak solution concept designed to rule out with confidence.

Definition 4 (Farsighted Stable Set) A set $V \subseteq Z$ is a farsighted stable set if

- for any $a, b \in V$ it is not the case that $a \gg b$, and

- for any $a \notin V$, there exists $b \in V$ with $b \gg a$.

The farsighted stable set is von Neumann and Morgenstern's [32] stable set defined with the indirect dominance relation. The first condition is known as internal stability, it requires no two stable states to indirectly dominate each other. The second condition is known as external stability, and it requires any unstable state to be indirectly dominated by a stable state.

${ }^{3}$ Our definition of dominance requires everybody in the coalition to be better off. An alternative definition of dominance would require the coalitions to move even if not everyone in the coalition is strictly better off. That is, one can say that $b$ dominates $a$ if there exists $S \subseteq N$ such that $a \stackrel{S}{\rightarrow} b, b \succeq_{i} a$ for all $i \in S$ and $b \succ_{j} a$ for some $j \in S$. Then one can appropriately modify all the succeeding definitions. This would significantly change our main solution concepts of the farsighted stable set and the largest consistent, but interestingly this would not affect our main result of equivalence and uniqueness of the farsighted stable set and the largest consistent set.

${ }^{4}$ Chwe [8] showed that a largest consistent set always exists, but it might be empty. 


\section{The Results}

\subsection{Motivation}

Both the largest consistent set and the farsighted stable set have been criticized for not incorporating the idea of maximality. This refers to the observation that under these solution concepts instead of considering the optimal course of action players form extreme expectations based on optimism (in the case of the farsighted stable set) or pessimism (in the case of the largest consistent set), which sometimes leads these solution concepts to make unreasonable predictions (see Greenberg [12], Ray and Vohra [28] and Xue [35]).

The example in Fig. 1 demonstrates this for the largest consistent set. In this example, the largest consistent set is $\{a, c, d\}$. $a$ is stable because under the assumptions of the largest consistent set, players are pessimistic and player 1 is afraid that player 2 will move to state $c$ following state $b$.

Similarly, the example in Fig. 2 demonstrates the problem for the farsighted stable set. The unique farsighted stable set is $\{c, d\}$. $a$ is not stable, because player 1 optimistically and unreasonably believes that player 2 will move to $c$ from state $b$.

The literature has proposed satisfactory modifications of these solution concepts that overcome the problem of maximality. For examples see Dutta and Vohra [11], Dutta and Vartiainen [10], Herings et al. [14], Karos and Robles [19], Kimya [20], and Konishi and Ray [23]. In this paper, we take a complementary approach and define conditions on abstract games under which the largest consistent set coincides with the farsighted stable set. This comes with additional benefits such as existence, uniqueness and the incorporation of the idea of maximality.

\subsection{Dominance Invariance and No Infinite Chains}

An abstract game satisfies Dominance Invariance when the dominance relation coincides with the indirect dominance relation.

Fig. 1 In this game, the largest consistent set is $\{a, c, d\}$, whereas the farsighted stable set is $\{c, d\}$
Fig. 2 In this game the largest consistent set is $\{a, c, d\}$, whereas the farsighted stable set is $\{c, d\}$
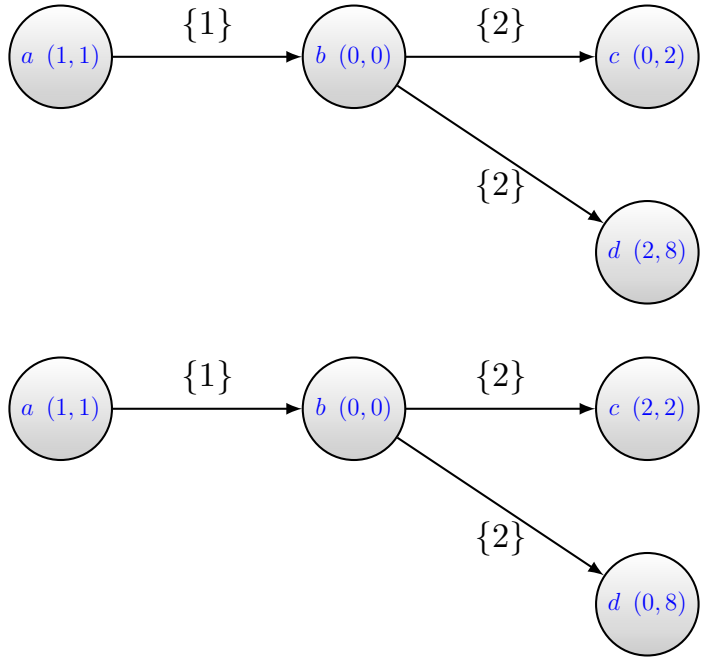
Fig. 3 Dominance Invariance is satisfied, but a farsighted stable set does not exist

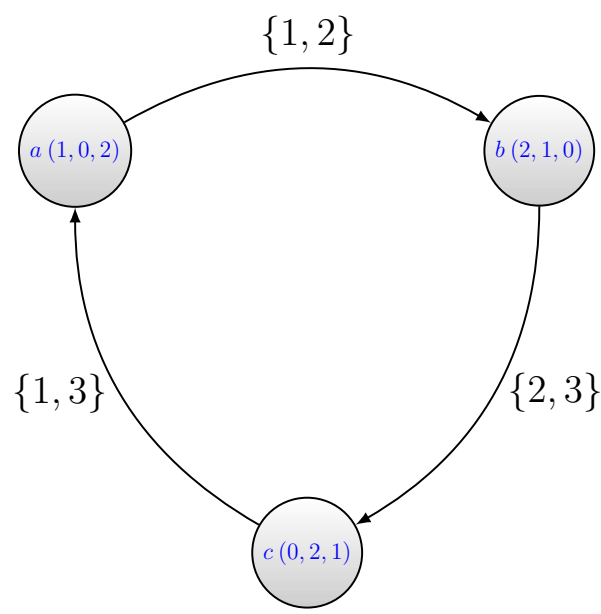

Definition 5 (Dominance Invariance) An abstract game $\Gamma$ satisfies Dominance Invariance if for any $a, b \in Z, a>b$ if and only if $a \gg b$.

One advantage of Dominance Invariance is that in these abstract games the farsighted solution concepts coincide with their myopic counterparts. For instance, the farsighted stable set coincides with the stable set, and the core coincides with the farsighted core.

This condition still does not imply the equivalence of the farsighted stable set and the largest consistent set. An example of this is the famous roommate problem modeled as a cycle (see Fig. 3). This abstract game satisfies dominance invariance, but the largest consistent set, which is $\{a, b, c\}$, does not coincide with the farsighted stable set. A farsighted stable set does not even exist in this game. ${ }^{5}$

The additional condition below ensures the equivalence between the farsighted stable set and the largest consistent set.

Definition 6 (No Infinite Chains) An abstract game $\Gamma$ satisfies No Infinite Chains if there is no $a_{1}, a_{2}, a_{3}, \ldots$ such that $a_{i}<a_{i+1}$ for all $i=1,2, \ldots$.

The game in Fig. 3 does not satisfy No Infinite Chains as the chain $a, b, c, a, b, c, \ldots$ is an infinite domination chain. The games in Figs. 1 and 2 do not satisfy Dominance Invariance. It turns out that in all games that satisfy both of these conditions we have the equivalence, existence and uniqueness of the farsighted stable set and the largest consistent set.

Chwe [8] showed that the largest consistent set always exists, but it might be empty and consequently it might also fail external stability. Chwe [8] also provided a condition under which the largest consistent set is non-empty and satisfies external stability. Xue [34] generalized Chwe's [8] condition considerably. It turns out that under Dominance Invariance and No Infinite Chains, Xue's [34] condition is satisfied implying the non-emptiness of the largest consistent set. ${ }^{6}$

5 The example can be modified to come up with a game that satisfies Dominance Invariance and in which both solution concepts exist, but differ. Hence, the problem here is not the non-existence of the farsighted stable set.

6 Chwe's [8] result is not enough to show the non-emptiness of the largest consistent set in our context. 
Lemma 1 If an abstract game satisfies Dominance Invariance and No Infinite Chains, then a unique non-empty largest consistent set exists that also satisfies external stability with both direct and indirect dominance relations. ${ }^{7}$

Proof Xue [34] showed that if there does not exist $a_{1}, a_{2}, \ldots$ such that $i<j$ implies $a_{j} \gg a_{i}$, then a unique nonempty largest consistent set exists and it satisfies external stability with the indirect dominance relation. Hence, all we need to do is to show that under Dominance Invariance and No Infinite Chains, Xue's [34] condition is satisfied.

Suppose $\Gamma$ satisfies both Dominance Invariance and No Infinite Chains. If there exists $a_{1}, a_{2}, \ldots$ such that $i<j$ implies $a_{j} \gg a_{i}$, then by Dominance Invariance there exists $a_{1}, a_{2}, \ldots$ such that $i<j$ implies $a_{j}>a_{i}$ and this in turn implies that there exists $a_{1}, a_{2}, \ldots$ with $a_{i+1}>a_{i}$ for all $i=1,2, \ldots$, which is a contradiction to No Infinite Chains. Hence, there does not exist $a_{1}, a_{2}, \ldots$ such that $i<j$ implies $a_{j} \gg a_{i}$.

The following lemma shows that if Dominance Invariance and No Infinite Chains are satisfied, then every consistent set satisfies internal stability.

Lemma 2 If an abstract game satisfies Dominance Invariance and No Infinite Chains, then each consistent set satisfies internal stability.

Proof Suppose $\Gamma$ satisfies Dominance Invariance and No Infinite Chains and suppose $V$ is a consistent set for $\Gamma$. Towards a contradiction, assume that $a_{2}, a_{1} \in V$ is such that $a_{2}>a_{1}$. Then there exists $S \subseteq N$ with $a_{1} \stackrel{S}{\rightarrow} a_{2}$ and $a_{2} \succ_{S} a_{1}$. By the consistency of $V$ there exists $a_{3} \in V$ with $a_{3} \gg a_{2}$ such that $a_{3} \nsucc_{S} a_{1}$. By Dominance Invariance, we also have that $a_{3}>a_{2}$.

But then we found $a_{3} \in V$ with $a_{3}>a_{2}$. Continuing to use the same argument, we can obtain a chain $a_{1}, a_{2}, a_{3}, \ldots$, where $a_{i+1}>a_{i}$ for all $i=1,2,3, \ldots$, which is a contradiction to No Infinite Chains. Hence, $V$ satisfies internal stability.

We are ready to prove the equivalence, existence and uniqueness of the farsighted stable set and the largest consistent set under Dominance Invariance and No Infinite Chains.

Proposition 1 If an abstract game satisfies Dominance Invariance and No Infinite Chains, then there exists a unique farsighted stable set that coincides with the largest consistent set.

Proof Let $\Gamma$ be a game that satisfies Dominance Invariance and No Infinite Chains. By Lemmas 1 and 2, there exists a non-empty and unique largest consistent set $V$ that satisfies both external and internal stability. Hence, $V$ is also a farsighted stable set.

Now, we will show that there can be no other farsighted stable set. Suppose $V^{\prime} \neq V$ is another farsighted stable set. By Proposition 3 of Chwe [8], each farsighted stable set is a subset of the largest consistent set. This implies that $V^{\prime} \subset V$. Take $a \in V \backslash V^{\prime}$. By the external stability of $V^{\prime}$, there exists $b \in V^{\prime} \subset V$ with $b \gg a$. This contradicts the internal stability of $V$, showing the uniqueness of the farsighted stable set.

We established the equivalence, existence and uniqueness of the two major solution concepts, but the proof does not hint at how to find this unique farsighted stable set and the largest consistent set. The proposition below establishes this with a simple recursive procedure when the game is finite.

${ }^{7}$ A set of states $V$ satisfies external stability with $>$ (with $\gg$ ) if for any $a \notin V$, there exists $b \in V$ with $b>a$ $(b \gg a)$. 
Proposition 2 Suppose $\Gamma$ is an abstract game that satisfies Dominance Invariance and No Infinite Chains. Let $f: 2^{Z} \rightarrow 2^{Z}$ be defined as $f(X)=\{a \in Z \mid$ there does not exist $b \in$ $X$ with $b>a$. Let $f^{1}(X)=f(X)$ and $f^{i+1}(X)=f\left(f^{i}(X)\right)$ for all $i=1,2, \ldots$ Then

- $f$ has a unique fixed point, which is the unique farsighted stable set and the largest consistent set, and

- if $Z$ is finite, then there exists $k^{*}>0$ such that $f^{k^{*}+1}(Z)=f^{k^{*}}(Z)$, i.e., if $Z$ is finite, then the unique farsighted stable set and the largest consistent set can be found by recursively applying $f$ starting from $Z$.

Proof $\emptyset$ cannot be a fixed point of $f$ and by Dominance Invariance any non-empty fixed point of $f$ is necessarily a farsighted stable set. This observation together with Proposition 1 implies that the unique fixed point of $f$ is the unique farsighted stable set.

To show the second statement assume that $Z$ is finite. $f^{1}(Z)$ is the set of all undominated states in $Z$. By No Infinite Chains, $f^{1}(Z) \neq \emptyset$. Note that for any odd integer $k>1$, $f^{k}(Z)=\left\{z \in Z \mid\right.$ for any $b \in Z$ with $b>z$, there exists $a \in \cup_{\{i<k \mid i \text { is odd }\}} f^{i}(Z)$ with $\left.a>b\right\}$. That is, for any odd integer $k>1, f^{k}(Z)$ is the set of states that are only dominated by some state that itself is dominated by some state in $\cup_{\{i<k \mid i \text { is odd }\}} f^{i}(Z)$.

This implies that $f^{i}(Z) \subseteq f^{i+2}(Z)$ for any odd $i$. Let $j$ odd be such that $f^{j}(Z)=$ $f^{j+2}(Z)$, which necessarily exists by the finiteness of $Z$.

Since $f^{j+1}(Z)$ is the set of states undominated by any state in $f^{j}(Z)$ and since $f^{j}(Z)$ satisfies internal stability, ${ }^{8}$ we have that $f^{j+1}(Z) \supseteq f^{j}(Z)$. Since $f^{j+2}(Z)=f^{j}(Z) \subseteq$ $f^{j+1}(Z)$ and $f^{j+2}(Z)$ is the set of states undominated by any state in $f^{j+1}(Z)$, we have that for any $z \in f^{j+1}(Z) \backslash f^{j+2}(Z)$ there exists $z^{\prime} \in f^{j+1}(Z) \backslash f^{j+2}(Z)$ such that $z^{\prime}>z$. If $f^{j+1}(Z) \backslash f^{j+2}(Z) \neq \varnothing$, then by the finiteness of $f^{j+1}(Z) \backslash f^{j+2}(Z)$, one can find an infinite dominance chain within $f^{j+1}(Z) \backslash f^{j+2}(Z)$, which would be a contradiction to No Infinite Chains. Hence, $f^{j+1}(Z) \backslash f^{j+2}(Z)=\emptyset$ implying that $f^{j+1}(Z)=f^{j+2}(Z)$.

Note that $f^{1}(Z) \subseteq f^{3}(Z) \subseteq f^{5}(Z) \subseteq \cdots$, where $f^{1}(Z)$ is the set of undominated states and whenever $k$ is odd $f^{k}(Z)$ is simply the set of states that are only dominated by some state that itself is dominated by some state in $\cup_{\{i<k \mid i \text { is odd }\}} f^{i}(Z)$.

Hence, the procedure outlined in Proposition 2 is equivalent to the intuitive inductive procedure in which one (i) labels the undominated states as stable, (ii) removes the set of states that are dominated by a state, which has already been labeled as stable, (iii) takes the set of undominated states from the remaining set and also labels them as stable, and (iv) gets back to step ii and repeats the procedure until a fixed point is reached.

\subsection{Maximality}

Both the farsighted stable set and the largest consistent set have been criticized for the problem of maximality (see Sect. 3.1 for examples and a more detailed description of this problem). In this section, we will show that another advantage of the conditions of Dominance Invariance and No Infinite Chains is that in domains satisfying these conditions farsighted stable set and the largest consistent set do not suffer from the problem of maximality.

For our argument, we use the (S)REFS developed in Dutta and Vohra [11] and show that under our assumptions if a (S)REFS exists, then it coincides with the farsighted stable set and the largest consistent set. (S)REFS assigns all agents an expectation that either deems a state stable or that defines a farsighted objection path to the state. It further imposes the

8 Remember that $f^{j}(Z)=\left\{z \in Z \mid\right.$ for any $b \in Z$ with $b>z, \exists a \in \cup_{\{i<j \mid i \text { is odd }\}} f^{i}(Z)$ with $\left.a>b\right\}$. 
properties that (i) it is indeed optimal for any coalition moving along an assigned farsighted objection path to take the particular move assigned by the expectation, and (ii) no coalition has an incentive to move out of a stable state given the expectation.

We formalize the definition now. An expectation function $E: Z \rightarrow Z \times 2^{N}$ is $E(x)=$ $(e(x), S(x))$, where $e(x)$ is the state that is expected to follow $x$ and $S(x)$ (with $x \stackrel{S(x)}{\longrightarrow} e(x)$ ) is the coalition expected to implement this change. Given an expectation $E(\cdot)=(e(\cdot), S(\cdot))$, let $e^{1}(x)=e(x)$ and $e^{i+1}(x)=e\left(e^{i}(x)\right)$ for all $i=1,2, \ldots$ An expectation $E(\cdot)=(e(\cdot), S(\cdot))$ is absorbing if for every $x \in Z$, there exists a $k^{*}$ such that $e^{k^{*}}(x)=e^{k^{*}+1}(x)$. In this case we let $e^{*}(x)=e^{k^{*}}(x)$, where $e^{k^{*}}(x)$ is stationary.

Definition 7 [(S)REFS (Dutta and Vohra [11])] An absorbing expectation $E$ is said to be a rational expectation if it satisfies $(I),(E)$ and $(M)$ below. If an absorbing expectation also satisfies $\left(M^{\prime}\right)$, then it is said to be strongly rational.

(I) If $x$ is stationary, then there does not exist $S, y$ such that $x \stackrel{S}{\rightarrow} y$ and $e^{*}(y) \succ_{S} x$.

(E) If $x$ is not stationary, then $e^{*}(x) \succ_{S(x)} x$.

$(M)$ If $x$ is not stationary, then there does not exist $y$ such that $x \stackrel{S(x)}{\longrightarrow} y$ and $e^{*}(y) \succ_{S(x)}$ $e^{*}(x)$.

$\left(M^{\prime}\right)$ If $x$ is not stationary, then there does not exist $T, y$ with $T \cap S(x) \neq \emptyset$ such that $x \stackrel{T}{\rightarrow} y$ and $e^{*}(y) \succ_{T} e^{*}(x)$.

The set of stationary points of a rational expectation is said to be a REFS. The set of stationary points of a strongly rational expectation is said to be a SREFS.

The first condition requires no coalition to have a profitable move at a stationary state. The second condition requires the move taken at a non-stationary state to make the coalition better off at the eventual terminal state. Condition $(M)$ requires the move taken by a coalition at a non-stationary state to be optimal in the sense that the coalition does not have another move that would make everybody in the coalition better off. REFS is the stationary points of an expectation that satisfies these three conditions. The definition of SREFS is obtained by replacing $(M)$ with the stronger maximality condition of $\left(M^{\prime}\right)$, which allows a coalition $T$ that has a nonempty intersection with $S(x)$ to be able to make a transition at $x$. Since this condition is harder to satisfy than the corresponding condition in REFS, every SREFS is necessarily a REFS.

REFS and SREFS deal with the problem of maximality by directly incorporating the optimality of coalitional moves in their definitions. Hence, if we can establish that the farsighted stable set and the largest consistent set are also a (S)REFS, then we would be demonstrating that these solution concepts also do not suffer from the problem of maximality. This is exactly what the next proposition does. It shows that under Dominance Invariance and No Infinite Chains, if (S)REFS exists then it is equivalent to the farsighted stable set and the largest consistent set.

Proposition 3 Suppose $\Gamma$ satisfies Dominance Invariance and No Infinite Chains. Furthermore, suppose that a (S)REFS exists. Then (S)REFS is unique and it is equivalent to the farsighted stable set and the largest consistent set.

Proof Suppose that $\Gamma$ satisfies Dominance Invariance and No Infinite Chains and also assume that a (S)REFS exists in $\Gamma$. Let $V$ be a (S)REFS. By the definition of a (S)REFS, $V$ satisfies internal stability with the direct dominance relation and external stability with the indirect dominance relation. By Dominance Invariance, (S)REFS satisfies both internal and external 
stability conditions with the indirect dominance relation implying that it is a farsighted stable set. Since each SREFS is a farsighted stable set and since farsighted stable set is unique and equivalent to the largest consistent set, we have that (S)REFS is also unique and equivalent to both the farsighted stable set and the largest consistent set.

\section{Two Classes of Games}

Dominance Invariance and No Infinite Chains together imply many desirable properties of the farsighted stable set and the largest consistent set such as existence, uniqueness, equivalence and respecting the notion of maximality. Given these strong implications, it should not come as a surprise that these are strong conditions. This section aims to show that there are still many interesting situations that can be modeled with abstract games that satisfy both of these conditions and in which all of the results in this paper apply.

We provide two simple classes of games, where one immediately satisfies No Infinite Chains and the other satisfies Dominance Invariance. From the literature, we give examples of situations that can be modeled with an abstract game that fall into each of these classes of games and that also satisfy both of our conditions.

\subsection{Finite Acyclic Games}

A state $b \in Z$ is reachable from state $a \in Z$, denoted $b R a$, if there exists a sequence of coalitions $S_{1}, S_{2}, \ldots, S_{k}$ and a sequence of states $a_{0}=a, a_{1}, a_{2}, \ldots, a_{k}=b$ such that $a_{i} \stackrel{S_{i+1}}{\longrightarrow} a_{i+1}$ for all $i=0,1, \ldots, k-1$.

Definition 8 (Finite Acyclic Games) $\Gamma$ is a finite acyclic game if $Z$ is finite and for any $a, b \in Z$ it is not the case that $a R b$ and $b R a$.

There are many situations that can be modeled as a finite acyclic game such as coalition formation when the decision to form or join a coalition is binding (see Bloch [5] and Ray and Vohra [27]), network formation when the decision to form a link is binding (see Aumann and Myerson [3]) and conflict (see Example 1 below).

Since dominance requires reachability, in any finite acyclic game No Infinite Chains condition is trivially satisfied. This means that on this domain we just need to check Dominance Invariance to see if the findings in this paper apply.

We first note that when Dominance Invariance is also satisfied, the unique farsighted stable set and the largest consistent set can be found with the simple inductive procedure in Proposition 4 below.

For any finite acyclic game $\Gamma$ let $Z_{\Gamma}=\left\{Z_{0}, \ldots, Z_{k^{*}}\right\}$ be the partition of $Z$ obtained in the following way. Let $Z_{0}$ denote the nodes from which no other node is reachable. By finiteness and acyclicity, $Z_{0} \neq \emptyset$. Let $Z_{1} \subseteq Z \backslash Z_{0}$ be the set of nodes from which only the nodes in $Z_{0}$ are reachable. Suppose $Z_{n}$ is defined for all $n \leq k$ then let $Z_{k+1} \subseteq Z \backslash\left\{\cup_{i=0,1, \ldots, k} Z_{i}\right\}$ be the set of nodes from which only the nodes in $\left\{\cup_{i=0,1, \ldots, k} Z_{i}\right\}$ are reachable.

Proposition 4 Let $\Gamma$ be a finite acyclic game that satisfies Dominance Invariance. Suppose $Z_{\Gamma}=\left\{Z_{0}, \ldots, Z_{k^{*}}\right\}$. Define $L_{0}=Z_{0}$ and $L_{k}=L_{k-1} \cup\left\{z \in Z_{k} \mid\right.$ there does not exist $z^{\prime} \in$ $L_{k-1}$ such that $\left.z^{\prime}>z\right\}$ for $k=1, \ldots, k^{*}$.

- $L_{k^{*}}$ is the unique farsighted stable set and the unique largest consistent set. 
Proof $L_{k^{*}}$ satisfies both internal and external stability and hence it is a farsighted stable set. Since the game satisfies both Dominance Invariance and No Infinite Chains, the farsighted stable set is unique and is equal to the largest consistent set.

The algorithm defined in Proposition 4 is the simple inductive algorithm in which (i) we label the terminal states as stable, (ii) we take all states from which only those states whose stability has been determined is reachable, (iii) among these we label a state as stable if only if it is not dominated by a state that is already labeled as stable, and (iv) we get back to (ii) and continue until all states are exhausted.

We finish this section with an example of a finite acyclic game that also satisfies Dominance Invariance.

Example 1 This example is modified from Acemoglu et al. [1]. ${ }^{9}$ There is a ruling coalition composed of $N$, where each individual is endowed with a political power $\gamma_{i} \in \mathbb{R}_{+}$. A coalition $S$ has sufficient power within $T \supseteq S$ if $\gamma_{S}=\sum_{i \in S} \gamma_{i}>\sum_{i \in T \backslash S} \gamma_{i}=\gamma_{T \backslash S}$.

If a coalition $S$ has sufficient power within $N$, then it might choose to eliminate $N \backslash S$ and collect the decision-making power in itself. But then any subset of $S$ with sufficient power within $S$ can eliminate the remaining players. This goes on until a self-enforcing ruling coalition $S^{*}$ emerges. Everybody prefers to be in the ultimate ruling coalition to being outside, and among the coalitions they belong to they prefer the ones with smaller total power.

This situation can be represented by an abstract game in which $Z$ includes the initial coalition $N$ and all coalitions that can possibly be reached from $N$. For any two states $S, T \in Z$ and coalition $F \subseteq N$, we have $S \stackrel{F}{\rightarrow} T$ if and only if $T=F \subseteq S$ and $\gamma_{T}>\gamma_{S \backslash T}$. Finally, for any two states $S, T \in Z$ and $i \in N, S \succ_{i} T$ if either $i \in S$ and $i \notin T$ or $i \in S \cap T$ and $\gamma_{S}<\gamma_{T}$. See Fig. 4 for the representation of the game when $N=\{1,2,3\}, \gamma_{1}=2$ and $\gamma_{2}=\gamma_{3}=1$.

Note that this game satisfies Dominance Invariance as all indirect dominance paths are necessarily of length one. Since the game is also a finite acyclic game, there exists a unique farsighted stable set in this game that coincides with the largest consistent set and that can be found with the simple inductive procedure defined in Proposition 4.

\subsection{Beeline Games}

Beeline games are finite games in which a path between any two states can always be replaced by a shortcut. More precisely, in beeline games whenever a state $y$ is reachable from a state $x$ through a path that can be initiated by $S$ then $S$ is also effective in changing the state from $x$ to $y$.

Definition 9 (Beeline Game) $\Gamma$ is a beeline game if $Z$ is finite and whenever $b \in Z$ is reachable from $a \in Z$ through $S$ (i.e., there exists $z_{1}, z_{2}, \ldots, z_{n}$ and $S_{1}, S_{2}, \ldots, S_{n-1}$ such that $z_{1}=a, z_{n}=b, S_{1}=S$ and $z_{i} \stackrel{S_{i}}{\rightarrow} z_{i+1}$ for all $\left.i=1, \ldots, n-1\right)$ we also have that $a \stackrel{S}{\rightarrow} b$.

Beeline games satisfy Domination Invariance. To see this suppose that $b \gg a$ and without loss of generality assume that $b$ indirectly dominates $a$ through an indirect dominance path

9 Also see Dutta and Vohra [11] for an analysis of this game with (S)REFS. Indeed, here they remark on the property of Dominance Invariance and the equivalence of the various solution concepts. 


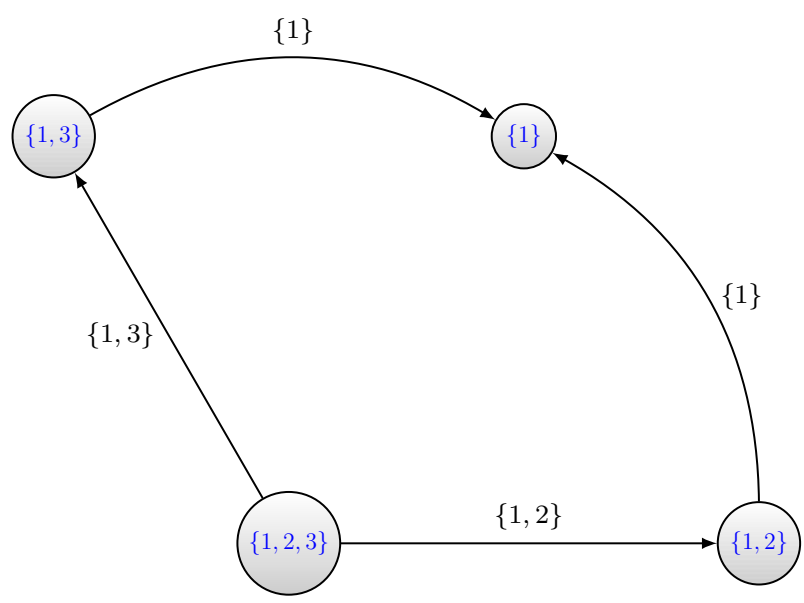

Fig. 4 Example 1 when $N=\{1,2,3\}, \gamma_{1}=2$ and $\gamma_{2}=\gamma_{3}=1$

initiated by $S$. Then the definition of beeline games requires $S$ to be able to enforce $b$ directly from $a$, implying $b>a$. Since Dominance Invariance is always satisfied in Beeline Games, we only need to check No Infinite Chains to see if the results of this paper apply.

Proposition 5 If $\Gamma$ is a beeline game that satisfies No Infinite Chains, then there exists a unique farsighted stable set that coincides with the unique largest consistent set.

Proof We already showed that beeline games satisfy Dominance Invariance, which means that the result follows by Proposition 1.

An immediate corollary of Propositions 4 and 5 is that in acyclic beeline games all of the results in this paper apply.

Corollary 1 If $\Gamma$ is an acyclic beeline game, then there exists a unique farsighted stable set in $\Gamma$ that coincides with the largest consistent set.

We finish with two examples of beeline games.

Example 2 This example is taken from Acemoglu et al. [2]. There is a finite set of states $\mathbb{S}$ and associated with each state $s \in \mathbb{S}$ is a set of winning coalitions $\mathbb{W}_{s}$. At any state $s$, any coalition $S \in \mathbb{W}_{s}$ can choose a new state $t \in \mathbb{S} \backslash s$ to transition to. Following this, any $S \in \mathbb{W}_{t}$ can choose another state to move to. This continues until a stable state is reached. Utilities are defined over $\mathbb{S}$. See Fig. 5 for the representation of this game when $\mathbb{S}=\left\{s_{1}, s_{2}, s_{3}\right\}$, $\mathbb{W}_{s_{1}}=\{S\}, \mathbb{W}_{s_{2}}=\{T\}$ and $\mathbb{W}_{s_{3}}=\{F\}$

This is a beeline game as the winning coalitions can impose any other state from each state. Acemoglu et al. [2] further assume that for any sequence of states $s_{1}, s_{2}, \ldots s_{k} \in \mathbb{S}$ if $s_{j+1}>s_{j}$ for all $j=1, \ldots, k-1$, then $s_{1} \nsucc s_{k} .{ }^{10}$ By the finiteness of the set of states, this assumption implies No Infinite Chains. Hence, both Dominance Invariance and No Infinite Chains are satisfied and all of the results in this paper apply.

10 This is the first part of Assumption 2 in Acemoglu et al. [2] rewritten in the language of our paper. 
Fig. 5 Example 2 when

$\mathbb{S}=\left\{s_{1}, s_{2}, s_{3}\right\}, \mathbb{W}_{s_{1}}=\{S\}$,

$\mathbb{W}_{s_{2}}=\{T\}$ and $\mathbb{W}_{s_{3}}=\{F\}$
Fig. 6 Example 3 when $N=\{1,2,3\}$ and $F=\{1\}$
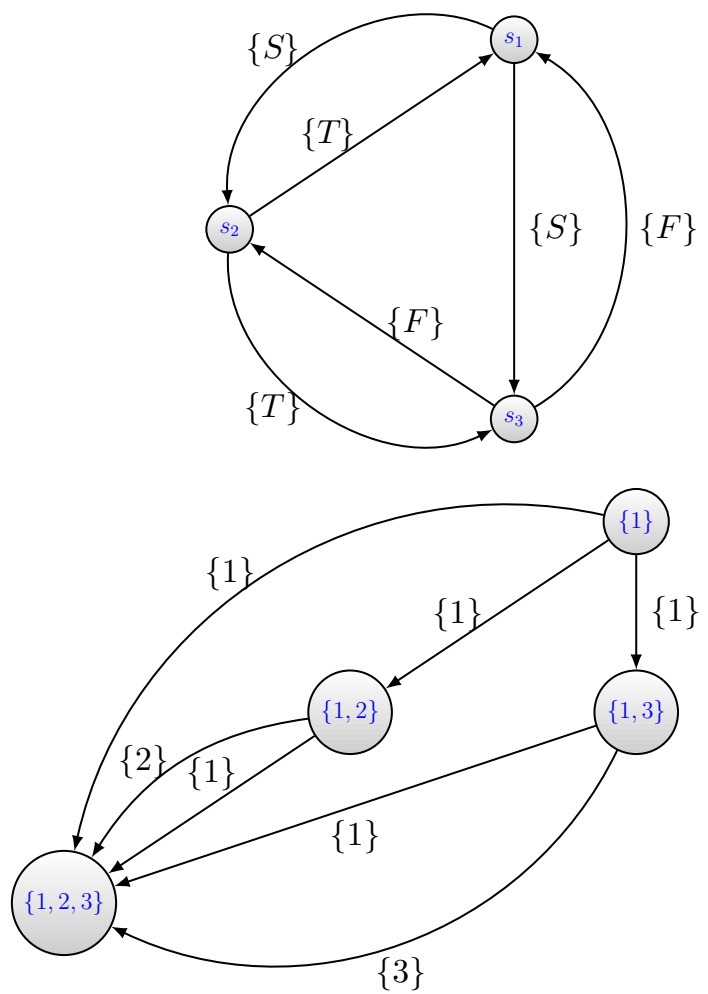

Example 3 This example is modified from Barbera et al. [4]. There is a society $N$ and a club $F \subseteq N$. Everybody in the society wants to be part of the club. Any member of the club $i \in F$ can admit new members $S \subseteq N \backslash F$ to the club. Once the new members are admitted, any member of the expanded club can admit any set of individuals that are not a part of the club. This continues until a stable club forms. Utilities are defined over the set of all possible clubs. ${ }^{11}$ See Fig. 6 for the representation of this game when $N=\{1,2,3\}$ and $F=\{1\}$.

This is a beeline game; furthermore, it is a finite acyclic game. Hence, it satisfies both Dominance Invariance and No Infinite Chains. This means that all of the results in this paper apply.

\section{Concluding Remarks}

\subsection{TU Games}

We have seen that Dominance Invariance and No Infinite Chains are conditions with very strong implications. Although we have also seen examples from the literature that satisfy these conditions, it shouldn't come as a surprise that these are indeed very strong conditions in certain domains.

11 In their model there is a fixed number of periods in which admission decisions can be made. We assume there is no such restriction. 
In this section, we demonstrate this using TU games and show that these conditions can never be satisfied in interesting TU Games.

A TU Game is a pair $(N, v)$, where $N$ is a finite set of players and $v: 2^{N} \rightarrow \mathbb{R}_{+}$is a function that associates a real number to every subset of $N$. We assume that $v(\emptyset)=v(i)=0$ for all $i \in N$ and $v$ is monotonic in the sense that $v(S) \geq v(T)$ whenever $S \supseteq T$.

We follow the approach in Ray and Vohra [29] in translating a TU Game into an abstract game. A state $x$ is a pair $(u(x), \pi(x))$, where $\pi(x)$ is a partition of $N$ and $u(x) \in \mathbb{R}^{N}$ is such that for all $S \in \pi(x), \sum_{i \in S} u_{i}(x)=v(S)$.

The effectiveness can be defined in several ways (see Ray and Vohra [29] for a detailed discussion). We will only be requiring the following two conditions, which are modified from Ray and Vohra [29] and Dutta and Vohra [11]. ${ }^{12}$

1. If $x \stackrel{T}{\rightarrow} y$, then $T \in \pi(y)$ and $S \backslash T \in \pi(y)$ for all $S \in \pi(x)$.

2. For every state $x \in Z, T \subseteq N$, and $w \in \mathbb{R}^{T}$ such that $\sum_{i \in T} w_{i}=v(T)$, there exists $y \in Z$ such that $x \stackrel{T}{\rightarrow} y, T \in \pi(y)$, and $u(y)_{T}=w$.

The first condition requires that the formation of a coalition $T$ cannot affect the structure of unrelated coalitions, and if $T$ includes some members of an existing coalition, then the residual remains intact. The second condition requires deviating coalitions to freely choose their own payoff as long as it is feasible for it. See Ray and Vohra [29] and Dutta and Vohra [11] for a more detailed discussion of these conditions.

Finally, we say that a TU Game is essential if $v(N)>0$. Observe that there is no interest in studying inessential TU Games as in these games the worth of every coalition is equal to 0 . The Proposition below establishes that Dominance Invariance cannot be satisfied in essential TU games.

Proposition 6 Suppose $\Gamma$ is an essential TU Game. Then $\Gamma$ cannot satisfy Dominance Invariance.

Proof Take any essential TU Game $\Gamma$. Let $S$ be a coalition with $v(S)>0$ and such that $v(T)=0$ for all $T \subset S$. Such a coalition $S$ exists, because $\Gamma$ is essential. Note that $|S| \geq 2$ as $v(i)=0$ for all $i \in N$.

Pick $k, j \in S$ with $k \neq j$. Let $X$ be the set of all states $x$ such that $S \in \pi(x), u_{k}(x)=\frac{3}{4} v(S)$ and $u_{t}(x)=\frac{1}{4(|S|-1)} v(S)$ for all $t \in S$ with $t \neq k$. Similarly, let $Y$ be the set of all states $y$ such that $S \in \pi(y), u_{j}(y)=\frac{3}{4} v(S)$ and $u_{t}(y)=\frac{1}{4(|S|-1)} v(S)$ for all $t \in S$ with $t \neq j$.

Observe that by Condition (2) on effectiveness, for any $x \in X$ there exists a state $x_{1}$ such that $x \stackrel{j}{\rightarrow} x_{1}$, where $\{j\} \in \pi\left(x_{1}\right)$ and $u_{j}\left(x_{1}\right)=0$. Furhermore, by Condition (1) we have that $u_{t}\left(x_{1}\right)=0$ for all $t \in S$. Then by Condition (2) there exists an indirect dominance path from $x$ to some $y \in Y$ in which first player $j$ moves from $x$ to $x_{1}$ and then $S$ moves from $x_{1}$ to $y$. Hence, $y \gg x$. But, it cannot be the case that $y>x$, because $S \in \pi(y), S \in \pi(x)$ and among $S$ only $j$ is better off as we move from $x$ to $y$.

Since $y \gg x$ and $y \ngtr x, \Gamma$ cannot satisfy Dominance Invariance.

Harsanyi [13] originally defined indirect dominance by requiring the coalitions moving along the indirect dominance path to be better off not only at the final outcome, but also in every transition. Hence, Harsanyi's [13] indirect dominance is different and a lot more demanding than Chwe's [8] indirect dominance definition. The latter became standard in the literature.

12 Our conditions are slightly weaker than what Dutta and Vohra [11] assume when analyzing simple games. 
Harsanyi [13] also defined absolutely stable games as the games in which Harsanyi's [13] indirect dominance relation is equivalent to direct dominance. ${ }^{13}$ Interestingly, Proposition 6 stands in great contrast to Weber's [33] characterization of absolutely stable games, according to which simple games are absolutely stable. ${ }^{14}$

Although Proposition 6 starkly demonstrates the limits of Dominance Invariance, it is still a condition of great interest, because (i) it implies farsighted solution concepts to be equivalent to their myopic counterparts, (ii) together with No Infinite Chains it implies the existence and uniqueness of the farsighted stable set and its equivalence to the largest consistent set, (iii) together with No Infinite Chains it also implies that both the farsighted stable set and the largest consistent set respect the idea of maximality, and finally (iv) as demonstrated in Sect. 4, even though it is strong condition, there is a range of situations of interest in which it is satisfied.

\subsection{Other Solution Concepts}

The equivalence of the farsighted stable set and the largest consistent set under Dominance Invariance and No Infinite Chains might seem to suggest that these two conditions completely eliminate the multiplicity of solution concepts in the farsighted stability literature, but this is not the case. An example of a prominent solution concept in the literature that would still not be equivalent to the unique farsighted stable set is DEM farsighted stable sets (see Herings et al. $[15,16])$.

Definition 10 (DEM Farsighted Stable Set) A set $V \subseteq Z$ is a DEM farsighted stable set if,

(D) for any $a \in V, b \notin V, S \subseteq N$ such that $a \stackrel{S}{\rightarrow} b$, there exists $c \in V$ with $c \gg b$ and $c \nsucc_{s} a$,

(E) for any $a \notin V$, there exists $b \in V$ with $b \gg a$,

$(M)$ there does not exist $V^{\prime} \subset V$ such that $V^{\prime}$ satisfies both $(D)$ and $(E)$.

Under Dominance Invariance and No Infinite Chains, the unique farsighted stable set is a DEM farsighted stable set, but there might exist others.

Proposition 7 Suppose $\Gamma$ satisfies Dominance Invariance and No Infinite Chains. Then the unique farsighted stable set is a DEM farsighted stable set, but there might exist other DEM farsighted stable sets.

Proof Take any $\Gamma$ that satisfies Dominance Invariance and No Infinite Chains. Let $V^{*}$ be the unique farsighted stable set. Since $V^{*}$ satisfies external stability, it also satisfies $(E)$. Furthermore by the equivalence of the farsighted stable set and the largest consistent set, $V^{*}$ is a consistent set, which implies that $(D)$ is also satisfied. Since any $V^{\prime} \subset V^{*}$ would violate external stability, $V^{*}$ also satisfies $(M)$ and hence $V^{*}$ is a DEM farsighted stable set.

To show that the other direction might not hold, consider the abstract game in which $N=\{1,2\}, Z=\{a, b, c\}, b \succ_{1} a \succ_{1} c, c \succ_{2} b \succ_{2} a, a \stackrel{1}{\rightarrow} b$, and $b \stackrel{2}{\rightarrow} c$. This game

\footnotetext{
13 Hence, absolute stability is the direct counterpart of Dominance Invariance. The former assumes the equivalence of dominance and Harsanyi's [13] indirect dominance, whereas the latter assumes the equivalence of dominance and Chwe's [8] indirect dominance.

14 Weber [33] does not impose the coalitional sovereignty conditions on the effectiveness relation. But, even when we impose these additional conditions on the effectiveness, absolutely stable games would still include simple games.
} 
satisfies both Dominance Invariance and No Infinite Chains. The unique farsighted stable set is $V^{*}=\{a, c\}$, but $V=\{b, c\}$ is a DEM farsighted stable set that is not a farsighted stable set.

Funding Open Access funding enabled and organized by CAUL and its Member Institutions.

Open Access This article is licensed under a Creative Commons Attribution 4.0 International License, which permits use, sharing, adaptation, distribution and reproduction in any medium or format, as long as you give appropriate credit to the original author(s) and the source, provide a link to the Creative Commons licence, and indicate if changes were made. The images or other third party material in this article are included in the article's Creative Commons licence, unless indicated otherwise in a credit line to the material. If material is not included in the article's Creative Commons licence and your intended use is not permitted by statutory regulation or exceeds the permitted use, you will need to obtain permission directly from the copyright holder. To view a copy of this licence, visit http://creativecommons.org/licenses/by/4.0/.

\section{References}

1. Acemoglu D, Egorov G, Sonin K (2008) Coalition formation in non-democracies. Rev Econ Stud 75(4):987-1009

2. Acemoglu D, Egorov G, Sonin K (2012) Dynamics and stability of constitutions, coalitions, and clubs. Am Econ Rev 102(4):1446-76

3. Aumann R, Myerson R (1988) Endogenous formation of links between players and coalitions: an application of the Shapley Value. In: Roth A (ed) The Shapley Value, Cambridge University Press, pp 175-191

4. Barbera S, Maschler M, Shalev J (2001) Voting for voters: a model of electoral evolution. Games Econ Behav 37(1):40-78

5. Bloch F (1996) Sequential formation of coalitions in games with externalities and fixed payoff division. Games Econ Behav 14(1):90-123

6. Bloch F, van den Nouweland A (2020) Farsighted stability with heterogeneous expectations. Games Econ Behav 121:32-54

7. Cai X, Kimya M (2021) Stability of alliance networks. Mimeo

8. Chwe MSY (1994) Farsighted coalitional stability. J Econ Theory 63(2):299-325

9. Diamantoudi E, Xue L (2003) Farsighted stability in hedonic games. Soc Choice Welf 21(1):39-61

10. Dutta B, Vartiainen H (2020) Coalition formation and history dependence. Theor Econ 15(1):159-197

11. Dutta B, Vohra R (2017) Rational expectations and farsighted stability. Theor Econ 12(3):1191-1227

12. Greenberg J (1990) The theory of social situations: an alternative game-theoretic approach. Cambridge University Press, Cambridge

13. Harsanyi JC (1974) An equilibrium-point interpretation of stable sets and a proposed alternative definition. Manag Sci 20(11):1472-1495

14. Herings PJJ, Mauleon A, Vannetelbosch VJ (2004) Rationalizability for social environments. Games Econ Behav 49(1):135-156

15. Herings PJJ, Mauleon A, Vannetelbosch VJ (2009) Farsightedly stable networks. Games Econ Behav 67(2):526-541

16. Herings PJJ, Mauleon A, Vannetelbosch VJ (2010) Coalition formation among farsighted agents. Games 1(3):286-298

17. Herings PJJ, Mauleon A, Vannetelbosch VJ (2019) Stability of networks under horizon-K farsightedness. Econ Theory 68(1):177-201

18. Herings PJJ, Mauleon A, Vannetelbosch VJ (2020) Matching with myopic and farsighted players. J Econ Theory 190:105125

19. Karos D, Robles L (2021) Full farsighted rationality. Games Econ Behav 130:409-424

20. Kimya M (2020) Equilibrium coalitional behavior. Theor Econ 15(2):669-714

21. Kimya M (2021) Farsighted objections and maximality in one-to-one matching problems. Mimeo

22. Kirchsteiger G, Mantovani M, Mauleon A, Vannetelbosch V (2016) Limited farsightedness in network formation. J Econ Behav Organ 128:97-120

23. Konishi H, Ray D (2003) Coalition formation as a dynamic process. J Econ Theory 110(1):1-41

24. Mauleon A, Vannetelbosch VJ, Vergote W (2011) von Neumann Morgenstern farsightedly stable sets in two-sided matching. Theor Econ 6(3):499-521 
25. Mauleon A, Molis E, Vannetelbosch VJ, Vergote W (2014) Dominance invariant one-to-one matching problems. Int J Game Theory 43(4):925-943

26. Page FH Jr, Wooders MH, Kamat S (2005) Networks and farsighted stability. J Econ Theory 120(2):257269

27. Ray D, Vohra R (1999) A theory of endogenous coalition structures. Games Econ Behav 26(2):286-336

28. Ray D, Vohra R (2014) Coalition formation. Handbook of Game Theory, vol 4. Elsevier, pp 239-326

29. Ray D, Vohra R (2015) The farsighted stable set. Econometrica 83(3):977-1011

30. Ray D, Vohra R (2019) Maximality in the farsighted stable set. Econometrica 87(5):1763-1779

31. Rosenthal RW (1972) Cooperative games in effectiveness form. J Econ Theory 5:88-101

32. Von Neumann J, Morgenstern O (1944) Theory of games and economic behavior. Princeton University Press, Princeton

33. Weber RJ (1976) Absolutely stable games. Proc Am Math Soc 55(1):116-118

34. Xue L (1997) Nonemptiness of the largest consistent set. J Econ Theory 73(2):453-459

35. Xue L (1998) Coalitional stability under perfect foresight. Economic Theory 11(3):603-627

Publisher's Note Springer Nature remains neutral with regard to jurisdictional claims in published maps and institutional affiliations. 